\title{
Clinical Effects of Torasemide Prolonged Release in Mild-to-Moderate Hypertension: A Randomized Noninferiority Trial Versus Torasemide Immediate Release
}

\author{
Alex Roca-Cusachs, ${ }^{1}$ Joaquín Aracil-Vilar, ${ }^{2}$ Carlos Calvo-Gómez, ${ }^{3}$ José-Vicente Vaquer-Pérez, ${ }^{4}$ \\ Félix Laporta-Crespo, ${ }^{5}$ María-José Rojas-Serrano, ${ }^{6}$ Antonio Guglietta, ${ }^{6}$ Savion Gropper, ${ }^{6}$ \\ and the Torasemide-PR in Hypertension Clinical Trial Investigators Group \\ 1 Unit of Hypertension, Department of Internal Medicine, Hospital de la Santa Creu i Sant Pau, Universitat Autònoma de Barcelona, Barcelona, Spain \\ 2 Centro de Salud El Cristo, Álvaro Florez Estrada 21, Oviedo, Spain \\ 3 Hospital Universitario de Santiago, Unidad HTA Edificio CCEE, C/ Choupana s/n, Santiago de Compostela, Spain \\ 4 Centro de Salud Petrel, Cura Jesús Zaragoza 3, Petrel, Alicante, Spain \\ 5 Centro de Salud La Roda, Mártires 6, La Roda, Albacete, Spain \\ 6 Pharmaceutical Research and Development Center, Ferrer Internacional, S.A., Barcelona, Spain
}

Keywords

Diuretic; Hypertension; Toresamide;

Toresamide prolonged released.

\section{Correspondence}

Alex Roca-Cusachs, MD, PhD, Unit of Hypertension, Department of Internal Medicine, Hospital de la Santa Creu i Sant Pau, Sant Antoni M. Claret 167, E-08025 Barcelona, Spain. Tel.: +34-93-2919342; Fax: +34-93-5565938;

E-mail: aroca@hsp.santpau.es

doi: 10.1111/j.1527-3466.2008.00046.x
The efficacy of a new torasemide prolonged release (PR) formulation to torasemide immediate release (IR) was compared in a randomized noninferiority double-blind trial. Patients with newly diagnosed mild-to-moderate hypertension or unresponsive or poor tolerability to previous antihypertensive monotherapy received $5 \mathrm{mg} /$ day of torasemide-PR $(\mathrm{n}=219)$ or torasemideIR $(\mathrm{n}=223)$ for 12 weeks (uptitration to $10 \mathrm{mg} /$ day if no response at 4 or 8 weeks). Mean diastolic blood pressure (DBP) reduction in the torasemidePR group (11.6 $\pm 7.1 \mathrm{mmHg}, 95 \%$ confidence interval [CI] 10.6-12.5) versus torasemide-IR (11.3 $\pm 7.5 \mathrm{mmHg}, 95 \%$ CI 10.2-12.3) met the noninferiority criterion of a nonsided $97.5 \%$ CI lower than the preestablished margin of $2 \mathrm{mmHg}$. A significantly higher percentage of patients in the torasemide-PR group achieved adequate BP control after 8 and 12 weeks. Ambulatory 24-h BP monitoring (ABPM) measurements in a subset of 100 patients showed greater daytime SBP reductions in the torasemide-PR group (128.4 $\pm 9.9 \mathrm{mmHg}$ vs. $133.5 \pm 10.4 \mathrm{mmHg}, P<0.05)$. Safety and tolerability of both formulations were similar.

\section{Introduction}

The ultimate goal of hypertension treatment requires a sufficient reduction in blood pressure (BP), and strict goals for target BP have been established (McVeigh et al. 1995). However, despite the existence of clear evidencebased guidelines (Chobanian et al. 2003; Mancia et al. 2007; Whitworth 2003), it is widely acknowledged that hypertension remains inadequately controlled, with only a small proportion of patients achieving target BP

For details of the Torasemide-PR in Hypertension Clinical Trial Investigators Group, please see the appendix.

Trial registration: EudraCT 2004-000876-14.

At the time of submission of the article, torasemide-PR has been approved by the Spanish Health authorities. levels (Turbull 2005). Oral diuretics have been the most popular step-one drug for the treatment of hypertension for more than 15 years. Diuretics have many of the attributes of the ideal step-one drug: they are inexpensive, easy to titrate, effective in a large percentage of patients with mild-to-moderate hypertension, well tolerated, and they enhance the effectiveness of other antihypertensive drugs (Gifford 1984).

Although thiazide diuretics have been the mainstay of treatment thus far, they continue to engender debate because of putative, undesirable side effects (Fukuda and Kimura 2006). Torasemide, a high-ceiling loop diuretic, has the advantage over older compounds to be effective at once-daily dose in the treatment of essential hypertension (Dunn et al. 1995; Friedel and Buckley 1991). High-ceiling (loop) diuretics exert their action 
by inhibiting the sodium reabsorption mechanisms of the thick ascending limb of the loop of Henle, thus resulting in substantial excretion of urine. Torasemide shows powerful diuretic and natriuretic actions with smaller potassium losses than equivalent doses of other loop diuretics like furosemide, which may be explained by a possible inhibitory action of torasemide on aldosterone activity (Dunn et al. 1995). Doses of torasemide of $5 \mathrm{mg}$ once daily are sufficient for antihypertensive treatment; these low doses of torasemide exert comparable antihypertensive effects to those of the overtly natriuretic doses of thiazides (e.g., hydrochlorothiazide $25 \mathrm{mg}$ ) (Baumgart 1993). In contrast to thiazides, torasemide does not cause significant renal potassium loss at doses recommended to treat hypertension (Luft 1993). Long-term antihypertensive treatment with torasemide has not resulted in undesired metabolic side effects, such as hypomagnesemia, alterations in glucose and lipid metabolism, or hyperuricemia (Baumgart 1993). A new torasemide prolonged release (PR) formulation has recently been developed. In two pharmacokinetic studies comparing both torasemide-PR and the already available immediate release (IR) formulation (Gropper et al. 2006a, 2006b), both formulations showed similar systemic exsposures represented by area under the curve (AUC) values within the bioequivalence 90\% confidence interval (CI) acceptance criteria (90\% CI 0.80-1.25), although torasemide-PR had proportionally higher AUCs than torasemide-IR. High maximum plasma concentrations $\left(C_{\max }\right)$ were significantly lower with torasemidePR compared with torasemide-IR and time to peak $\mathrm{C}_{\max }$ $\left(t_{\max }\right)$ was significantly longer with torasemide-PR than with torasemide-IR.

According to European Medicines Agency (EMEA) Guidelines for modified release formulations (The European Agency for the Evaluation of Medicinal Products 1999), a randomized noninferiority clinical trial was designed to compare the antihypertensive efficacy of this new formulation of torasemide-PR to the already available IR formulation of the same drug in the treatment of patients with mild-to-moderate essential hypertension. Safety and tolerability of torasemide-PR were also evaluated.

\section{Patients and Methods}

\section{Study Design}

A randomized, double-blind, two-arm, parallel-group, multicenter study was designed to demonstrate the noninferiority of the antihypertensive efficacy of torasemidePR in comparison to torasemide-IR in patients with mildto-moderate essential hypertension. The duration of the study was 3 months. The study took place from April 2005 to February 2006 in two countries in Europe (Spain and Russia) and involved the participation of hypertension units of 41 centers.

The study protocol was approved by the institutional review board of each of the participating centers and was conducted in accordance with the principles of the Declaration of Helsinki and its amendments. Written informed consent was obtained from all participants prior to enrolment in the study.

\section{Eligibility}

Male and female patients of any race, aged between 18 and 75 years, with newly diagnosed mild-tomoderate hypertension, defined as systolic BP (SBP) 140$179 \mathrm{mmHg}$ and diastolic BP (DBP) 90-109 mmHg according to the 2003 European Society of HypertensionEuropean Society of Cardiology guidelines (European Society of Hypertension-European Society of Cardiology Guidelines Committee 2003) or previously treated patients with monotherapy who did not respond or did not tolerate his/her current medication were eligible for the trial. Exclusion criteria were: history of unresponsiveness to diuretic monotherapy or need to use combination therapy to achieve BP control effectively; secondary hypertension or severe hypertension; myocardial infarction or stroke in the preceding 6 months; unstable angina, heart failure, major arrhythmia, or conduction disturbance; type 1 and type 2 diabetes mellitus; significant renal or hepatic dysfunction; obesity (body mass index [BMI] $\geq$ $40 \mathrm{~kg} / \mathrm{m}^{2}$ ); concurrent use of aspirin, nonsteroidal antiinflammatory drugs (NSAIDs), classes Ia, Ib, and II antiarrhythmic drugs, lithium, etacrinic acid, or aminoglycoside antibiotics; known hypersensitivity to the study medication or intolerance to lactose, as well as any contraindication for prescribing treatment with torasemide considered by the investigator. Pregnant women, nursing mothers, or women of childbearing potential not using adequate methods of contraception were also excluded.

\section{Treatment and Patient Evaluation}

Antihypertensive medication of previously treated patients was discontinued or tapered off gradually before the start of the run-in period. After a 2-week placebo run-in, all eligible patients were sequentially assigned to one of the two masked medications according to a predetermined computer-generated randomization schedule. Patients were randomized ( $1: 1)$ to torasemide-PR or torasemide-IR (Sutril ${ }^{\circledR}$; Novag-Ferrer, Barcelona, Spain) $5 \mathrm{mg}$, taken in the morning after fasting overnight before eating or drinking. The study medication was dispensed 
to patients at each visit to cover the period of time until the next visit.

Causal readings of SBP and DBP were taken with OMRON M7 BP monitor with the patient in the sitting position after $5 \mathrm{~min}$ of rest. Patients were instructed to attend clinical visits without taking the study medication. Moreover, patients were advised to avoid alcohol, cigarette smoking, coffee/tea, and exercise for at least 30 min before BP measurement. Ambulatory 24-h BP monitoring (ABPM) was carried out in a subgroup of patients at the end of the run-in period before the first drug administration and on the last day of treatment. A previously validated noninvasive automatic device (Spacelabs 90217; Spacelabs, Inc., Redmond, WA, USA), preset at 20-min intervals, was used. Measurements were taken at the nondominant arm. Patients were instructed to adhere to their normal daily activities and regular sleeping hours. For analysis, daytime episodes were defined from 10:00 until 22:00, and night time episodes from 24:00 until 06:00. Variables recorded were as follows: mean SBP, DBP, mean arterial pressure (MAP), heart rate (HR), and pulse pressure (PP) for 24-h, daytime, and night time periods. Additionally, reductions of SBP and DBP 4-h postdose compared to casual readings before starting ABPM procedure were measured. Recordings with more than $20 \%$ erroneous readings and those who did not have at least one valid record per hour were excluded from the final analysis.

Assessments were performed before the run-in period, at baseline (visit 1), 4 weeks (visit 2), 8 weeks (visit 3), and 12 weeks (visit 4) after initiation of the treatment with torasemide. At visit 2 or visit 3, the dose of torasemide-IR or -PR could be uptitrated to $10 \mathrm{mg}$ if the decrease in DBP was lower than $10 \%$ of the baseline value. Patients with a DBP decrease of at least $10 \%$ compared to baseline or with a DBP value $<90 \mathrm{mmHg}$ received the same dose $(5 \mathrm{mg}$ or $10 \mathrm{mg}$ ) of torasemide throughout the study. Patients with inadequate control of BP at visit 3, that is, after 4 weeks of treatment with torasemide $5 \mathrm{mg}$ and 4 weeks with torasemide $10 \mathrm{mg}$, were withdrawn from the study. These patients were included in the efficacy analysis.

At each visit, BP and HR were measured, the use of concomitant medication was recorded, compliance with treatment was checked, and patients were interviewed for urinary symptoms and the occurrence of adverse events. Noncompliance was defined as taking less than $75 \%$ of the prescribed course of medication. All adverse events were designated by the investigator as either drug related or not drug related. Patients were asked for time of onset, duration, and intensity of the adverse event. The intensity was determined by subjective evaluation of the patient and classified as mild, moderate, and severe.
At the end of the run-in phase and at the end of treatment, each patient underwent a complete medical examination that included a 12-lead electrocardiogram (ECG) and complete laboratory studies.

\section{Efficacy and Safety Parameters}

The primary efficacy variable was the decrease in casual DBP achieved at the end of the treatment period compared to baseline values. Secondary efficacy variables included the decrease in casual SBP achieved at the end of treatment compared to baseline and the percentage of patients with adequate control of BP at the end of the study, defined as SBP $<140 \mathrm{mmHg}$ and/or DBP $<90 \mathrm{mmHg}$. In the subset of patients with ABPM, antihypertensive efficacy of the study medication was assessed by differences in the mean 24-h SBP and DBP, mean daytime and night time SBP and DBP, and circadian variability at the end of treatment compared to baseline. Tolerability and safety parameters were the incidence and severity of adverse events reported throughout the study as well as changes in vital signs, clinical laboratory tests, 12-lead ECG, and the presence of urinary symptoms, including the percentage of patients with urinary symptoms, such as urinary urgency (frequency and intensity) and nocturia (frequency). Treatment compliance was also recorded.

\section{Statistical Analysis}

Statistical analyses included: (1) the per-protocol (PP) population for all randomized patients who had completed the study; (2) the PP population with missing data replaced by last observation carried forward (LOCF) imputation method for missing endpoint values at visit 4 (PP-LOCF); (3) the full analysis set (FAS) population for all patients who were randomized whether or not they took medication; (4) the FAS population with LOCF imputation for missing endpoint values at visit 4 (FASLOCF); (5) the ABPM population for all patients who completed ABPM at the end of the run-in period and on the last day of treatment measurement; and (6) the safety population for all patients who were randomized and received at least one administration of the study drug. All randomized patients without protocol violations, who received 3 months of treatment, attended the study visits during 3 months, and presented a compliance of treatment of $\geq 75 \%$, were considered evaluable for efficacy. Patients who were withdrawn after 2 months of treatment due to lack of response to the dose of $10 \mathrm{mg}$ were also considered evaluable for efficacy. The primary efficacy analysis was performed in the PP-LOCF population of evaluable patients. 


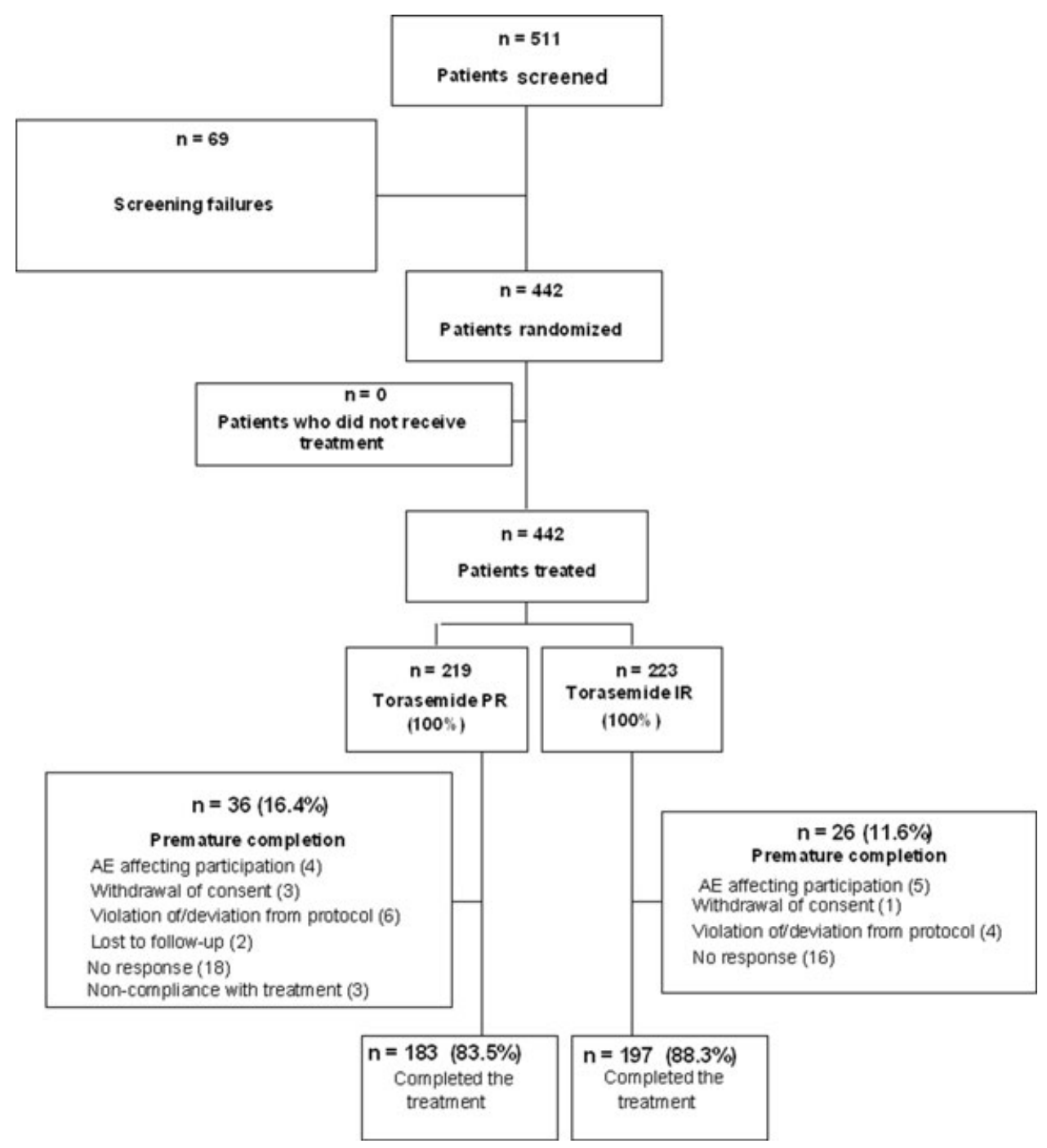

Figure 1 Flow-chart of the study population.

The sample size calculation was based on a noninferiority study approach. Assuming a pooled standard deviation (SD) of $7 \mathrm{mmHg}$ and a $97.5 \%$ confidence interval (CI) for one-sided test with a noninferiority margin (delta) of $2 \mathrm{mmHg}$ in the DBP readings between the two treatment groups, a sample size of 194 evaluable patients in each group (total 388) was calculated to provide a power of $80 \%$.

The mean changes in $\mathrm{BP}$ from baseline to the final value were compared using an analysis of covariance (ANCOVA), including the baseline value as covariate. Repeated measures analysis of variance (ANOVA) was used to assess differences in mean values of ABPM variables between the two treatment groups. Categorical variables were compared with the chi-square $\left(\chi^{2}\right)$ test or the Fisher exact test and continuous variables with the Mann-Whitney $U$-test. All hypothesis were tested with a
0.05 probability of a type 1 error. Double-data entry was carried out with a subsequent validation to guarantee the quality and consistency of the data. SAS software version 8.2 (SAS Institute, Inc., Cary, NC, USA) was used for the analysis of data.

\section{Results}

\section{Study Population}

Of a total of 511 patients who were recruited by 41 investigators, 442 fulfilled the eligibility criteria and were randomized to treatment with torasemide-PR $(n=219)$ or torasemide-IR $(n=223)$. A flow-chart of the study population is shown in Figure 1. The FAS-LOCF population included 201 patients in the torasemide-PR group and 213 in the torasemide-IR group, and the PP population included 183 in the torasemide-PR group and 197 in 
Table 1 Baseline characteristics of the study population (full analysis set population).

\begin{tabular}{|c|c|c|}
\hline Data & Torasemide-PR & Torasemide-IR \\
\hline Total patients & 219 & 223 \\
\hline \multicolumn{3}{|l|}{ Sex, no. (\%) } \\
\hline Men & $76(34.7)$ & $93(41.7)$ \\
\hline Women & $143(65.3)$ & $130(58.3)$ \\
\hline Age, years, mean (SD) & $54.8(11.5)$ & $55.1(11.1)$ \\
\hline Weight, kg, mean (SD) & $77.6(13.2)$ & $79.1(12.8)$ \\
\hline $\mathrm{BMI}, \mathrm{kg} / \mathrm{m}^{2}$, mean (SD) & $29.2(4.4)$ & $29.2(4.2)$ \\
\hline \multicolumn{3}{|l|}{ Race, no. (\%) } \\
\hline Caucasian & $217(99.1)$ & $222(99.6)$ \\
\hline Black & $1(0.5)$ & $1(0.5)$ \\
\hline Others & $1(0.5)$ & \\
\hline Current smokers, no. (\%) & $38(17.3)$ & 55 (24.7) \\
\hline Alcohol use, no. (\%) & $24(10.9)$ & $27(12.1)$ \\
\hline \multicolumn{3}{|l|}{ Medical history, no. (\%) } \\
\hline Heart disease & $12(5.5)$ & $11(4.9)$ \\
\hline Endocrine disease & $12(5.5)$ & $13(5.8)$ \\
\hline Neoplasm & $21(9.9)$ & $21(9.4)$ \\
\hline Renal/urinary disease & $19(8.7)$ & $23(10.3)$ \\
\hline \multicolumn{3}{|l|}{$\begin{array}{l}\text { Reason for prescribing torasemide, } \\
\text { no. }(\%)\end{array}$} \\
\hline Newly diagnosed hypertension & 87 (39.7) & $83(37.2)$ \\
\hline $\begin{array}{l}\text { Inadequate BP control with previous } \\
\text { antihypertensive drugs }\end{array}$ & $108(49.3)$ & $121(54.3)$ \\
\hline $\begin{array}{l}\text { Intolerance to previous } \\
\text { antihypertensive drugs }\end{array}$ & $24(10.7)$ & $19(8.5)$ \\
\hline
\end{tabular}

the torasemide-IR group. Of a total of 130 patients undergoing ABPM, 65 assigned to torasemide-PR and 65 to torasemide-IR; 14 in the torasemide-PR arm and 16 in the torasemide-IR arm were excluded because of invalid recordings at the end of the run-in period and/or on the last day of treatment.

There were 169 men and 273 women, with a mean ( \pm SD) age of $54.9 \pm 11.3$ years, mean weight of $78.4 \pm$ $13.1 \mathrm{~kg}$, and mean BMI of $29.2 \pm 4.3 \mathrm{~kg} / \mathrm{m}^{2}$. Ninetynine percent of patients were Caucasian. Treatment with torasemide was given because of inadequate BP control with previous antihypertensive medication in $52 \%$ of patients, lack of tolerability in $9.7 \%$, and newly diagnosed hypertension in $39 \%$. Baseline characteristics of patients assigned to the group of torasemide-PR or to torasemideIR were similar as shown in Table 1.

\section{Efficacy}

After 12 weeks of treatment, patients assigned to treatment with torasemide-PR showed a mean reduction in DBP of $11.6 \pm 7.1 \mathrm{mmHg}$ (95\% CI 10.6-12.5) compared to $11.3 \pm 7.5 \mathrm{mmHg}(95 \%$ CI $10.2-12.3)$ in those as- signed to torasemide-IR (ANCOVA, $P=0.435$ ) (Fig. 2). In the torasemide-PR group, DBP decreased from a mean of $97.1 \pm 4.5 \mathrm{mmHg}(95 \%$ CI 96.4-97.7) at visit 1 to $85.5 \pm 7.1 \mathrm{mmHg}(95 \%$ CI 84.5-86.4) at visit 4, which was similar to a DBP decrease from a mean of $97.5 \pm$ $4.8 \mathrm{mmHg}(95 \%$ CI 96.9-98.2) at visit 1 to $86.3 \pm 6.8$ $\mathrm{mmHg}(95 \% \mathrm{CI} 85.4-87.2)$ at visit 4 in the torasemide-IR group (ANCOVA, $P=0.354$ ). The antihypertensive efficacy of both regimens was similar because the difference in mean changes in DBP between the torasemide-PR and torasemide-IR groups fulfilled the noninferiority criterion of a nonsided $97.5 \%$ CI lower than the preestablished margin of $2 \mathrm{mmHg}$. These results for the PP-LOCF data set were also confirmed in a sensitivity analysis carried out in the remaining PP, FAS, and FAS-LOCF populations (Fig. 3).

Changes in SBP after 12 weeks of treatment were also similar in both study arms, with a mean reduction of $17.3 \pm 11.2 \mathrm{mmHg}(95 \%$ CI 15.7-18.2) in the torasemide-PR group compared to $17.2 \pm 10.8 \mathrm{mmHg}$ (95\% CI 15.7-18.6) in the torasemide-IR group (ANCOVA, $P=0.713$ ) (Fig. 3). In the torasemide-PR group, SBP decreased from a mean of $154.5 \pm 9.8 \mathrm{mmHg}(95 \%$ CI 153.1-155.8) at visit 1 to $137.2 \pm 11.5 \mathrm{mmHg}(95 \%$ CI 135.6-138.8) at visit 4, which was similar to a SBP decrease from a mean of $155.2 \pm 9.7 \mathrm{mmHg}(95 \%$ CI $163.9-156.5)$ at visit 1 to $138.1 \pm 9.9 \mathrm{mmHg}(95 \% \mathrm{CI}$ 136.7-139.4) at visit 4 in the torasemide-IR group (ANCOVA, $P=0.579)$. Although treatment with torasemide$\mathrm{PR}$ and torasemide-IR were not significantly different in decreases in DBP and SBP obtained after 12 weeks of treatment, patients assigned to any formulation of the diuretic showed a statistically significant decrease in both DBP and SBP at the end of the study as compared to baseline values (Fig. 4).

The percentage of patients with adequate control of $\mathrm{BP}$, defined as SBP $<140 \mathrm{mmHg}$ and/or DBP $<90$ mmHg, was significantly higher among those who received torasemide-PR compared to torasemide-IR, both after 8 weeks $(69.4 \%$ vs. $58.4 \%$, Fisher exact test, $P=$ $0.025)$ and 12 weeks (63.9\% vs. 51.3\%, Fisher exact test, $P=0.031$ ) of treatment (Fig. 5). At the end of treatment (12 weeks) in the PP population, the relative effect size was of $25 \%$ higher percentage of patients controlled with torasemide-PR (63.9\% [117/183]) than with torasemideIR $(51.3 \%$ [101/197]) (relative risk 1.25, 95\% CI 1.0476$1.4845)$.

Baseline values in the subset of 100 patients who had evaluable ABPM measurements (torasemide-PR $\mathrm{n}=51$, torasemide-IR $\mathrm{n}=49$ ) are shown in Table 2 . There were no statistically significant differences between the torasemide-PR and torasemide-IR groups after 12 weeks of treatment in the mean 24-h SBP 


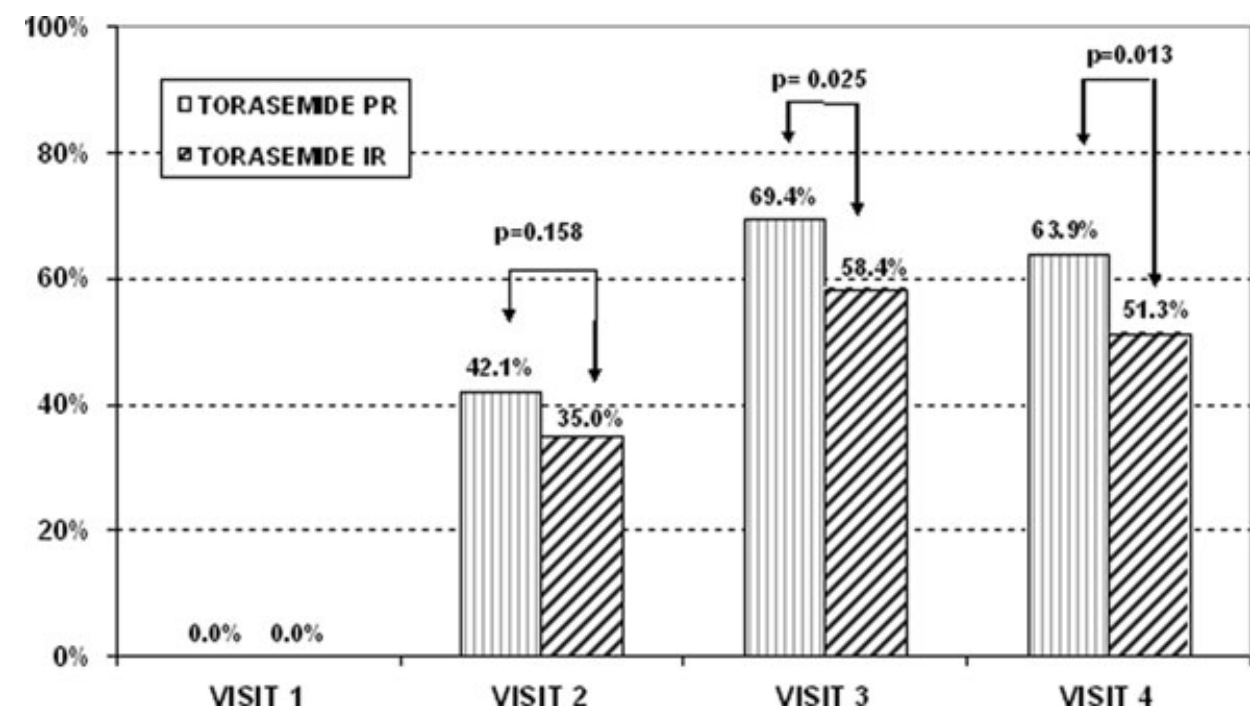

Figure 2 Sensitivity analysis of differences in DBP reduction between torasemide-PR and torasemide-IR after 12 weeks of treatment in the PP-LOCF, PP, FAS, and FAS-LOCF populations.

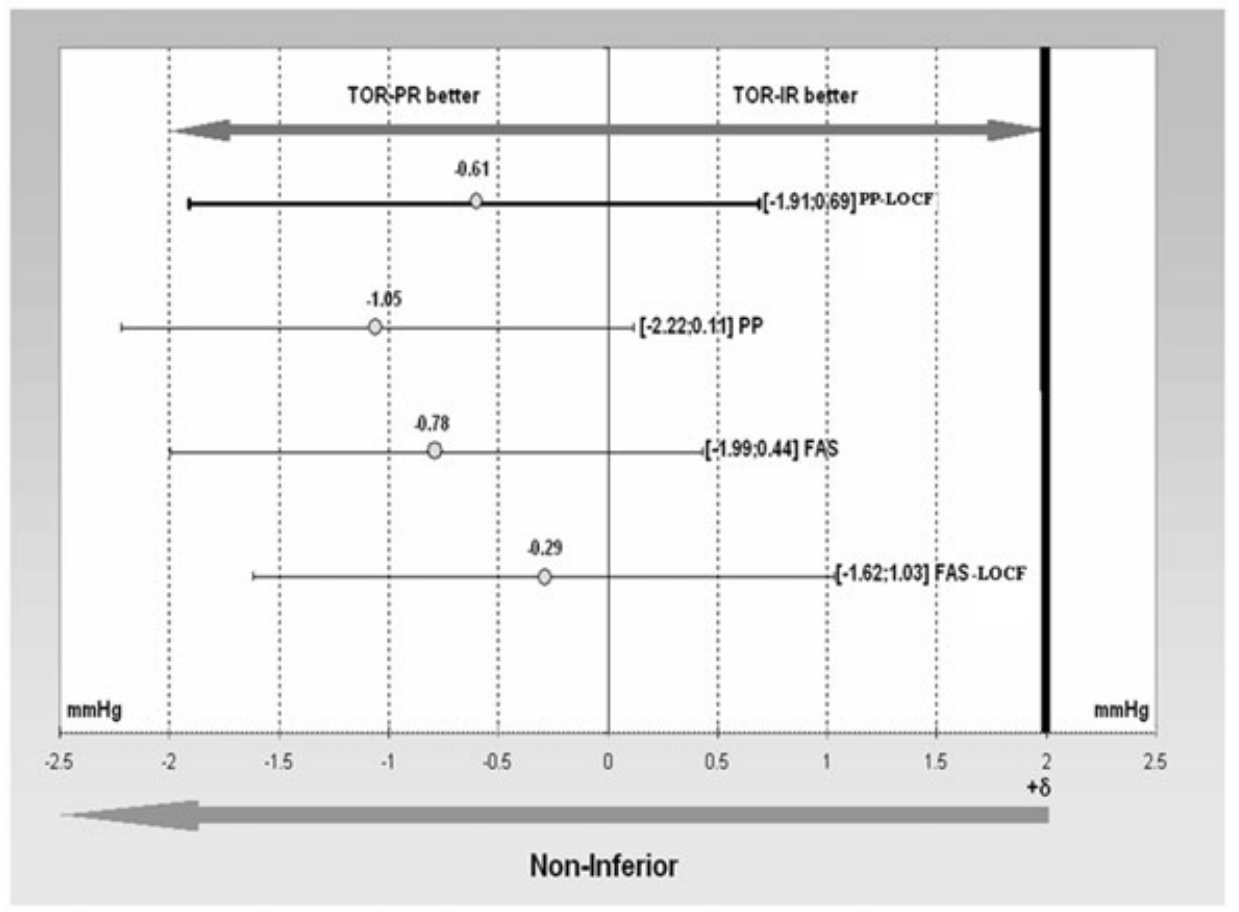

Figure 3 Mean changes in DBP and SBP from baseline to 12 weeks of treatment with torasemide-PR and torasemide-IR (PP-LOCF population).

$(125.2 \pm 10.1 \mathrm{mmHg}$ and $128.9 \pm 8.9 \mathrm{mmHg}$, respectively) (ANOVA, $P=0.051$ ) nor in the mean 24-h $\mathrm{DBP}(78.1 \pm 6.6$ and $80.5 \pm 7.6 \mathrm{mmHg}$, respectively) (ANOVA, $P=0.1$ ). In both study groups, night time SBP, DBP, and MAP values were significantly lower than daytime values $(P<0.001)$. Daytime SBP was significantly lower in the torasemide-PR group (128.4 \pm
9.9 $\mathrm{mmHg})$ compared to the torasemide-IR group (133.5 $\pm 10.4 \mathrm{mmHg}$ ) (ANOVA, $P=0.01$ ) (Table 3).

\section{Urinary Symptoms}

The percentage of patients with urinary urgency in the torasemide-PR and torasemide-IR groups was $47.0 \%$ and 


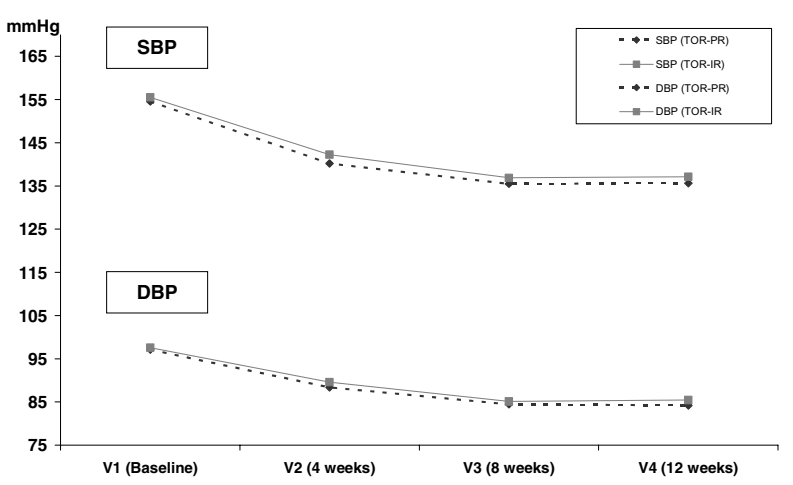

Figure 4 Decreases in SBP and DBP during the 12-week study period in both treatment arms (PP dataset).( $\square$ torasemide-PR, $\mathbf{\nabla}-$ - $\mathbf{t}$ torasemide-IR).

$51.2 \%$, respectively, at the end of the study. In the torasemide-PR group, $37.7 \%$ of patients presented more than five episodes of urinary urgency versus $43 \%$ of patients in the torasemide-IR group. In total, $24.1 \%$ of patients in the torasemide-PR group presented more than two episodes of urinary urgency versus $39.3 \%$ in the torasemide-IR group.

\section{Safety and Tolerability}

Eighty-five percent of patients treated with torasemidePR and $88 \%$ of those treated with torasemide-IR completed the 12-week study period. Doses of the study had to be increased from $5 \mathrm{mg}$ to $10 \mathrm{mg}$ in $49.5 \%$ of patients in the torasemide-PR group and in $55.3 \%$ of patients in the torasemide-IR group.

Adverse events were documented in 29.7\% (65/219) patients treated with torasemide-PR, with a total of 120 adverse events, and in $29.1 \%$ (65/223) of those given torasemide-IR, with a total of 114 adverse events. In more than $95 \%$ of cases, adverse events were of mild or moderate intensity. The most frequent adverse events were headache, palpitations, dizziness, fatigue, and nose bleeding. No serious drug-related adverse events were observed. The discontinuation rates due to adverse events were $1.8 \%(4 / 219)$ in patients treated with torasemide-PR and $2.2 \%(5 / 223)$ in patients treated with torasemide-IR. Vital signs, physical examination, and ECG measures showed no relevant alterations during the study. All patients showed laboratory blood parameters (including ions, glucose, and lipid profile) within normal range, or minor abnormalities were considered without any clinical relevance according to the investigator. There were no cases of new onset of diabetes or clinically relevant increases in cholesterol fractions or clinically relevant decrease in potassium levels.

\section{Discussion}

The objective of this study was to compare the efficacy of a PR formulation of torasemide to the standard

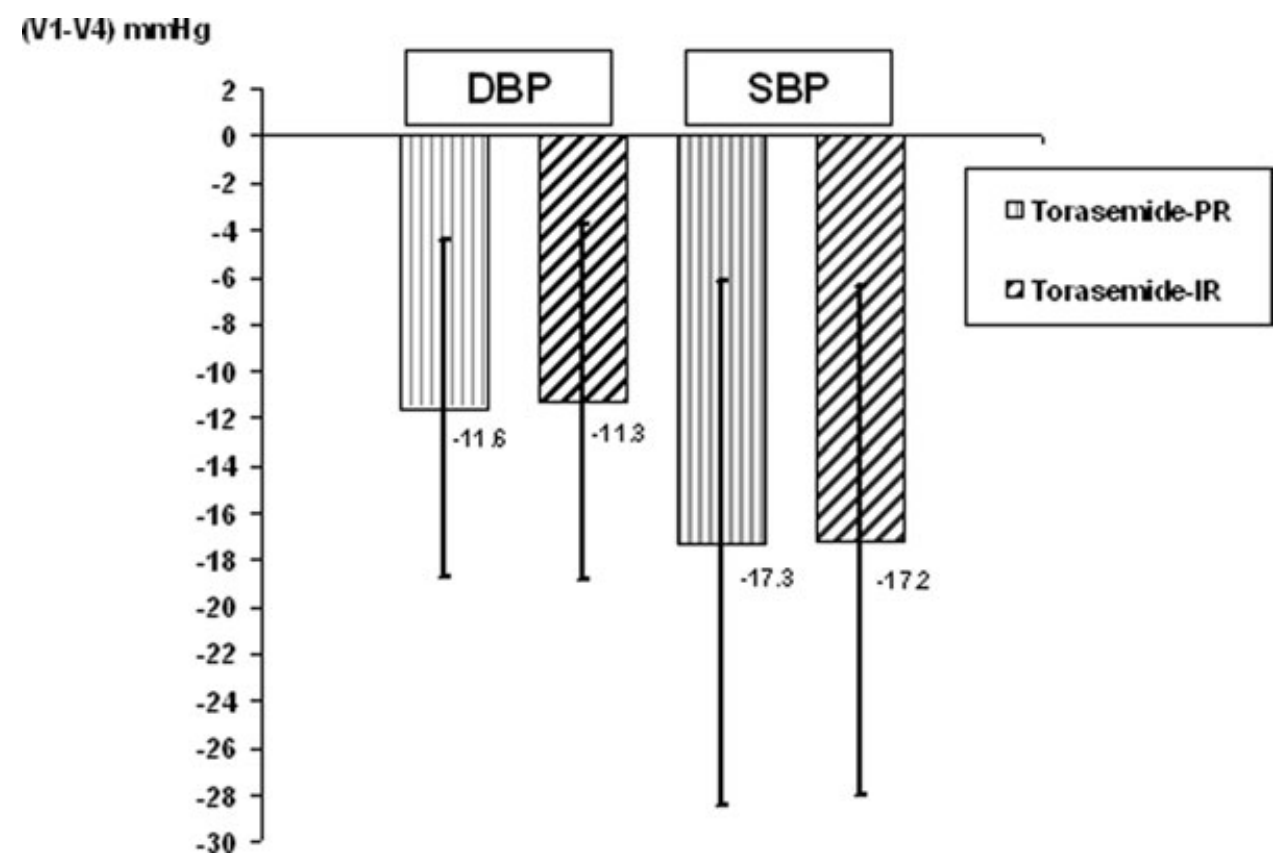

Figure 5 Percentages of patients with adequately controlled blood pressure during the study period (PP dataset). 
Table 2 ABPM: Mean SBP, DBP and MBP values at baseline in the Torasemide-PR and Torasemide-IR study groups.

\begin{tabular}{lcc}
\hline ABPM (mmHg) & $\begin{array}{c}\text { Torasemide-PR } \\
\text { Mean (SD) }\end{array}$ & $\begin{array}{c}\text { Torasemide-IR } \\
\text { Mean (SD) }\end{array}$ \\
\hline Daytime SBP & $139.45(12.71)$ & $141.79(9.88)$ \\
Night time SBP & $123.14(14.83)$ & $125.60(11.28)$ \\
Daytime DBP & $87.69(6.98)$ & $89.67(9.12)$ \\
Night time DBP & $73.08(7.68)$ & $74.26(8.40)$ \\
Daytime MBP & $101.47(8.03)$ & $103.49(6.50)$ \\
Night time MBP & $105.84(8.08)$ & $107.54(8.24)$ \\
\hline
\end{tabular}

torasemide-IR formulation in controlling mild-tomoderate essential hypertension. For this purpose, a parallel design was employed over a sample of patients providing adequate power and significance level to demonstrate the noninferiority of torasemide-PR versus torasemide-IR with a margin of $2 \mathrm{mmHg}$ of sitting DBP readings. The duration of the study (12 weeks) was sufficient to assess the efficacy and tolerability of antihypertensive monotherapy with torasemide-PR. Both treatments achieved significant and consistent reductions in SBP and DBP, supporting the external validity of the trial. The CIs for the difference between the treatment groups did not reach the noninferiority margin ( $2 \mathrm{mmHg}$ ), thus establishing the noninferiority of the test formulation (torasemide-PR) versus the active comparator (torasemide-IR).

Treatment groups obtained by random allocation were homogeneous regarding demographic characteristics and baseline status of the disease under investigation. The percentage of patients with a recent hypertension diagnosis was slightly higher in the torasemide-PR group than in the torasemide-IR group $(39.7 \%$ and $37.2 \%$, respectively), whereas the proportion of patients previously treated for hypertension was slightly higher in the torasemide-IR group than in the torasemide-PR group (54.3\% and $49.3 \%$, respectively). The use of a placebo run-in period excluded from randomization patients who responded to placebo.
At the end of the 12-week active treatment period, there were relevant and significant reductions in SBP and DBP in patients treated with torasemide-PR and torasemide-IR. Although these reductions were similar in both groups, the percentage of patients with adequate control of BP after 8 and 12 weeks of treatment was significantly higher in patients treated with the PR formulation. Other statistically significant differences in efficacy parameters were not observed except for significantly lower daytime SBP values in the torasemide-PR group compared to torasemide-IR in a subgroup of patients who had ABPM measurements. Both treatments were well tolerated. The tolerability profile regarding urinary symptoms agreed with the expected profile of these medications.

Approximately half of the patients required increasing the dose of study medication to control BP (49.5\% for the PR formulation and 55.3\% for the IR formulation). Many hypertensive patients will require more than one drug to achieve adequate control of BP and the management hypertension guidelines recommend optimizing dosages or adding additional drugs as a second step in the pharmacological approach of this condition. Diuretics are effective, well tolerated, easy to titrate, and comparatively inexpensive (Krakoff 2005; Salvetti and Ghiadoni 2006). Our results provide further evidence that torasemide is a first-line drug in the treatment of mild-to-moderate hypertension and that the option of increasing diuretic dose is a good therapeutic approach before adding another antihypertensive agent.

All these data suggest that torasemide-PR formulations offer a good BP response in mild or moderate hypertensive patients. It was also observed that torasemide-PR is superior in the degree of BP control and it also shows better SBP control as measured by ABPM recording. The superiority of ABPM to casual BP measurement in the prediction of cardiovascular risk and target organ damage is well established (Clement et al. 2003; Staessen et al. 2001).

Both formulations of torasemide showed good BP response. Torasemide-PR once daily at doses up to $10 \mathrm{mg}$

Table 3 ABPM: Mean SBP, DBP, and MBP values at visit 4: Torasemide-PR versus Torasemide-IR and daytime versus night time.

\begin{tabular}{|c|c|c|c|c|c|}
\hline ABPM (mmHg) & $\begin{array}{c}\text { Torasemide-PR } \\
\text { Mean (SD) }\end{array}$ & $\begin{array}{l}\text { Torasemide-IR } \\
\text { Mean (SD) }\end{array}$ & $\begin{array}{l}\text { PR vs. IR } \\
P \text { value }\end{array}$ & $\begin{array}{l}\text { PR daytime vs. night time } \\
\qquad P \text { value }\end{array}$ & $\begin{array}{l}\text { IR daytime vs. night time } \\
\qquad P \text { value }\end{array}$ \\
\hline Daytime SBP & $128.37(9.86)$ & $133.48(10.40)$ & 0.0187 & $<0.001$ & $<0.001$ \\
\hline Night time SBP & $115.42(12.21)$ & $115.54(10.51)$ & 0.9568 & & \\
\hline Daytime DBP & $82.24(6.62)$ & $84.92(9.33)$ & 0.0998 & $<0.001$ & $<0.001$ \\
\hline Night time DBP & $68.85(8.03)$ & $68.12(8.26)$ & 0.6530 & & \\
\hline Daytime MBP & $98.45(7.02)$ & $101.66(8.97)$ & 0.0521 & $<0.001$ & $<0.001$ \\
\hline Night time MBP & $85.55(8.62)$ & $84.77(8.14)$ & 0.6384 & & \\
\hline
\end{tabular}


during 12 weeks was found to have noninferior antihypertensive efficacy than torasemide-IR in a population of mild-to-moderate hypertensive patients. Adequate control of BP was obtained in a higher percentage of patients treated with the PR formulation either after 8 or 12 weeks of treatment and better response in 24-h ABPM recordings was observed. Both PR and IR formulations were well tolerated and showed similar safety and tolerability profiles.

\section{Acknowledgments}

We thank Marta León, BSc, Marta Llorens, RN, Elena Roldán, RN, Oscar Buhigas, BSc, and Ramón Dosantos, BSc, from Trial Form Support for clinical trial monitoring and statistical analysis; Julia V. Smolina, MD, Olga A. Erokhina, MD, and Daria V. Zvereva, MD, from PSI Pharma Support International for clinical monitoring; Ester Fernandez, MD, from the Medical Department of Ferrer Internacional, S.A., for medical review; and Marta Pulido, MD, for editing the manuscript and for editorial assistance.

\section{Conflict of Interest}

The authors have no conflict of interest.

\section{References}

Baumgart P (1993) Torasemide in comparison with thiazides in the treatment of hypertension. Cardiovasc Drugs Ther 7(Suppl 1):63-68.

Chobanian AV, Bakris GL, Black HR, Cushman WC, Green LA, Izzo JL Jr, Jones DW, Materson BJ, Oparil S, Wright JT Jr, Roccella EJ (2003) The Seventh Report of the Joint National Committee on Prevention, Detection, Evaluation, and Treatment of High Blood Pressure: The JNC 7 report. JAMA 289:2560-2572.

Clement DL, De Buyzere ML, De Bacquer DA, de Leeuw PW, Duprez DA, Fagard RH, Gheeraert PJ, Missault LH, Braun JJ, Six RO, Van Der Niepen P, O’Brien E (2003) Prognostic value of ambulatory blood-pressure recordings in patients with treated hypertension. N Engl J Med 348:2407-2415.

Dunn CJ, Fitton A, Brogden RN (1995) Torasemide. An update of its pharmacological properties and therapeutic efficacy. Drugs 49:121-142.

European Society of Hypertension-European Society of Cardiology Guidelines Committee (2003) 2003 European Society of Hypertension-European Society of Cardiology guidelines for the management of arterial hypertension. J Hypertens 21:1011-1053.

Friedel HA, Buckley MMT (1991) Torasemide. A review of its pharmacological properties and therapeutic potential. Drugs 41:81-103.
Fukuda M, Kimura G (2006) Pathophysiology of antihypertensive therapy with diuretics. Hypertens Res 29:645-653.

Gifford RW Jr (1984) The role of diuretics in the treatment of hypertension. Am J Med 77:102-106.

Gropper S, Albet C, Guglietta A, Antonijoan R, Gich I, Barbanoj MJ (2006a) Single-dose, randomized, cross-over bioavailability pilot clinical trial of torasemide immediate release compared to a new prolonged release formulation of torasemide [abstract]. Basic Clin Pharmacol Toxicol 99(Suppl 1):48.

Gropper S, Rojas MJ, Guglietta A, Pelagio P, Antonijoan R, Gich I, Barbanoj MJ (2006b) Single and repeated-dose, randomized, cross-over, bioavailability clinical trial of torasemide immediate release compared to a new prolonged release formulation of torasemide [abstract]. Basic Clin Pharmacol Toxicol 99(Suppl 1):48.

Krakoff LR (2005) Diuretics for hypertension. Circulation 112:127-129.

Luft FC (1993) Torasemide in the treatment of arterial hypertension. J Cardiovasc Pharmacol 22(Suppl 3):S32S39.

Mancia G, De Backer G, Dominiczak A, Cifkova R, Fagard R, Germano G, Grassi G, Heagerty AM, Kjeldsen SE, Laurent S, et al. (2007) 2007 Guidelines for the management of arterial hypertension: The Task Force for the Management of Arterial Hypertension of the European Society of Hypertension (ESH) and of the European Society of Cardiology (ESC). J Hypertens 25:1105-1187.

McVeigh GE, Flack J, Grimm R (1995) Goals of antihypertensive therapy. Drugs 49:161-175.

Salvetti A, Ghiadoni L (2006) Thiazide diuretics in the treatment of hypertension: An update. J Am Soc Nephrol 17:S25-S29.

Staessen JA, Asmar R, De Buyzere M, Imai Y, Parati G, Shimada K, Stergiou G, Redon J, Verdecchia P (2001) Task Force II: Blood pressure measurement and cardiovascular outcome. Blood Press Monit 6:355-370.

The European Agency for the Evaluation of Medicinal Products. Committee for Proprietary Medicinal Products (CPMP) (1999) Note for guidance on modified release oral and transdermal dosage forms: Section II (pharmacokinetic and clinical evaluation). CPMP/EWP/280/96 London.

Turbull F (2005) Managing cardiovascular risk factors: The gap between evidence and practice. PLoS Med 2:e131.

Whitworth JA, World Health Organization, International Society of Hypertension Writing Group (2003) 2003 World Health Organization (WHO)/International Society of Hypertension (ISH) statement on management of hypertension. J Hypertens 21:1983-1992.

\section{Appendix}

Torasemide-PR in Hypertension Clinical Trial Investigators Group (listed in alphabetical order): 
Nadezhda Alexeeva (St. Petersburg Cardiology Clínic, St. Petersburg, Russia); Pedro Aranda (Hospital Carlos Haya, Málaga, Spain); Josep Lluis Ballvé (CAP Florida Nord, Barcelona, Spain); Andrei Baranovsky (St. Petersburg City Hospital \#31, St. Petersburg, Russia); Svetlana Boldueva (St. Petersburg Mechnikov State Medical Academy, Department of Internal Diseases, St. Petersburg, Russia); Carles Brotons (CAP Sardeña, Barcelona, Spain); Angel Cano (CAP Bon Pastor, Barcelona, Spain); Svetlana Churina (St. Petersburg Pokrovskaya Hospital, St. Petersburg, Russia); Antoni Coca (Hospital Clínic i Provincial, Universitat de Barcelona, Barcelona, Spain); Luis De Teresa (Hospital Sant Vicenç de Raspeig, San Vicente de Raspeig, Alicante, Spain); Juan Antonio Divisón (CS de Fuentealbilla, Fuentealbilla, Albacete, Spain); Yanis Dumpis (St. Petersburg Mechnikov State Medical Academy, North West Center of Arrhythmia Diagnostics and Treatment, St. Petersburg, Russia); Xavier Farrés (CAP El Remei, Vic, Barcelona, Spain); Ángela Felip (Hospital de Mataró, Barcelona, Spain); Juan García (Hospital La Paz, Madrid, Spain); Blas Gil (Hospital Universitario San Cecilio, Granada, Spain); Boris Goloschekin (St. Petersburg City Hospital \#15, St. Petersburg, Russia); Pablo Gómez (Hospital General Jerez de La Frontera, Jerez de la Frontera, Cádiz, Spain); Victor Kostenko (St. Petersburg
City Outpatient Unit \#109, St. Petersburg, Russia); Enrique López de Coca (CS Chinchilla, Chinchilla, Albacete, Spain); Manuel Luque (Hospital Clínico San Carlos, Madrid, Spain); Emili Marco (CAP Sarrià de Ter, Girona, Spain); Joan Martorell (CS Guisona, Lleida, Spain); Viktor Milyagin (Smolensk State Medical Academy, Smolensk, Russia); Alexander Orlov-Morozov (Moscow City Hospital \#23, Moscow, Russia); Vladimir Parfenov (Moscow City Hospital \#61, Moscow, Russia); Elena Pavlysh (St. Petersburg City Outpatient Unit \#25, St. Petersburg, Russia); Jaume Plana (Hospital Residencia San Camilo, Sant Pere de Ribes, Barcelona, Spain); Miguel Ángel Prieto (CS Vallobín Concinos, Oviedo, Spain); Montserrat Pujol (CAP El Carmel, Barcelona, Spain); José Carlos Rodríguez (Hospital Dr. Negrín, Palmas de Gran Canaria, Spain); Alexander Sherenkov (Krasnogvardeysky District Medical-Physiology Clinic, St. Petersburg, Russia); Javier Sobrino (Hopsital del Esperit Sant, Santa Coloma de Gramanet, Barcelona, Spain); Lev Sorokin (St. Elizabeth City Hospital, St. Petersburg, Russia); Joaquim Vilà (Hospital Municipal de Badalona, Barcelona, Spain); Irina Zobenko (JSC Chernaya Rechka Health Center, St. Petersburg, Russia); and Konstantin Zrazhesvsky (St. Petersburg City Hospital \#8, St. Petersburg, Russia). 